\title{
Diversidade fúngica no ar de centros comerciais em Fortaleza, Ceará
}

\author{
Josiany Costa de Souza ${ }^{1}$ (D), Ane Teles Reis ${ }^{2}$, Jonas Felipe da Silva Araujo ${ }^{3}$ (D), \\ Karine Silva Pimentel ${ }^{3}$ D , Itatiaia Sampaio Souza ${ }^{3}$, Marcos Adelino Almeida \\ Filho $^{3}$ (D) Lydia Dayanne Maia Pantoja ${ }^{3}$ (D) \& Germana Costa Paixão ${ }^{3,4}$
}

(1) Instituto Federal de Educação, Ciência e Tecnologia do Ceará, Programa de Pós-Graduação em Tecnologia e Gestão Ambiental, Avenida Treze de Maio 2081, Fátima 60040-531, Fortaleza, Ceará, Brasil. E-mail: josiany.costa@aluno.uece.br

(2) Universidade Federal do Ceará, Faculdade de Medicina, Programa de Pós-Graduação em Ciências Médicas, Rua Coronel Nunes de Melo 1315, Rodolfo Teófilo 60430-270, Fortaleza, Ceará, Brasil. E-mail: aneteles@aluno.uece.br

(3) Universidade Estadual do Ceará - Campus do Itaperi, Centro de Ciências da Saúde, Avenida Dr. Silas Munguba 1700, Itaperi 60714-903, Fortaleza, Ceará, Brasil. E-mail: lydia.pantoja@uece.br

(4) Universidade Federal do Ceará - Campus do Porangabuçu, Faculdade de Medicina, Programa de PósGraduação em Microbiologia Médica, Rua Cel. Nunes de Melo 1315, Rodolfo Teófilo, 60430-275, Fortaleza, Ceará, Brasil. E-mail: germana.paixao@uece.br

Souza J.C., Reis A.T., Araujo J.F.S., Pimentel K.S., Souza I.S., Almeida Filho M.A., Pantoja L.D.M. \& Paixão G.C. (2019) Diversidade fúngica no ar de centros comerciais em Fortaleza, Ceará. Pesquisa e Ensino em Ciências Exatas e da Natureza, 3(2): 125-133. http://dx.doi.org/10.29215/pecen.v3i2.1258

Editora acadêmica: Anne Evelyne Franco de Souza. Recebido: 01 Julho 2018. Aceito: 06 Setembro 2019. Publicado: 02 Outubro 2019.

Resumo: A presente pesquisa investigou a diversidade fúngica no ar de dois centros comercias com climatização natural, sendo um centro comercial de gestão particular (CCPa) e outro de gestão pública $(\mathrm{CCPu})$, com intuito de analisar a qualidade de ar no ambiente construído. Realizou-se durante o período de um ano coletas mensais de ar, por meio da técnica de sedimentação passiva em placas de Petri para isolamento e contagem global de colônias de gêneros fúngicos anemófilos. Os dados climáticos foram fornecidos pela Fundação Cearense de Meteorologia e Recursos Hídricos. Foram isolados maior quantidade e diversidade de gêneros fúngicos no CCPa do que no CCPu. Os espaços com maior presença fúngica foram os estacionamentos e os andares térreos, devido a características peculiares como menor renovação do ar e presença de unidade alimentar. Os fungos filamentosos hialinos, representados pelos gêneros Acremonium, Aspergillus, Chrysonilia, Fusarium e Penicillium estiveram presentes em todos os ambientes, possivelmente motivados por sua distribuição ubíqua. Não foi possível estabelecer relação deles com as condições climáticas. A partir desses dados afirma-se a necessidade do monitoramento periódico da qualidade do ar em centros comerciais, podendo fornecer aos seus gestores medidas remediadoras, propondo manter o equilíbrio com o bem-estar das pessoas que usam/transitam no ambiente.

Palavras chave: Fungos, ambiente construído, qualidade do ar, Aerobiologia.

\section{Fungal dispersion in the air of commercial centers in Fortaleza, Ceará}

Abstract: The present research investigated the fungal diversity in the air of two commercial centers with natural climate, being a commercially managed shopping center (CCPa) and another of public management $(\mathrm{CCPu})$, with the purpose of analyzing the air quality in the environment built. Monthly collection of air was carried out during the period of one year by means of the passive sedimentation technique in Petri plates for isolation and global counting of colonies of anemophilic fungal genera. The climatic data were provided by the Cearense Foundation of Meteorology and Water Resources. A greater quantity and diversity of fungal genera were isolated in CCPa than in CCPu. The spaces with greater 
fungal presence were the parking lots and the ground floors, due to peculiar characteristics like less renovation of the air and presence of food unit. The hyaline filamentous fungi, represented by the genera Acremonium, Aspergillus, Chrysonilia, Fusarium and Penicillium were present in all environments, possibly motivated by their ubiquitous distribution. Their relationship with climatic conditions could not be established. Based on these data, it is necessary to regularly monitor air quality in commercial centers, and can provide its managers with remedial measures, aiming to maintain the balance with the well-being of people who use / transit in the environment.

Key words: Fungi, built environment, air quality, Aerobiology.

\section{Introdução}

A qualidade de vida da população é influenciada pela qualidade do ar, especialmente em grandes centros urbanos onde a concentração de pessoas é bem maior e em boa parte do tempo as pessoas ficam inseridas em ambientes fechados (Quadros 2008).

A característica de um ambiente interno relaciona-se diretamente à qualidade do ar externo, como também, as atividades realizadas dentro de edificações, como o fumo e vapores eliminados no cozimento de alimentos, o aquecimento de ar e água, ou mesmo os detritos e materiais de construção e móveis (Statholoupou et al. 2008; Sunyer et al. 2017).

Dessa maneira, a colonização do ar depende das condições do ambiente e conforme a área analisada, os micro-organismos variam em quantidade e qualidade, podendo estabelecer dois tipos de microbiota aérea: de ambientes abertos e de ambientes fechados (Pimentel et al. 2016).

No Brasil, adotam-se como metodologia para controle e padronização da Qualidade do Ar Interno (QAI) as resoluções e regulamentos técnicos da Agência Nacional de Vigilância Sanitária (Anvisa), em concordância com as normas técnicas da Associação Brasileira de Normas Técnicas (ABNT). Como parâmetros fundamentais sugeridos para avaliação e definidos pelas regulamentações são: umidade relativa, temperatura e velocidade do ar, fungos e bactérias, material particulado e dióxido de carbono (Basto 2007).

Os poluentes químicos e biológicos são considerados pela Organização Mundial da Saúde (OMS) como causadores da Síndrome do Edifício Doente (SED). Nesse cenário, a ventilação do ambiente é considerada ótima alternativa para proporcionar condições aceitáveis de ar no ambiente interno, ressaltando-se a dificuldade de controlar os poluentes e suas fontes de emissão (Schossler et al. 2015).

A SED consiste no desconforto causado a trabalhadores, pacientes ou usuários de ambientes internos, através de uma relação direta com a qualidade do ambiente construído que estão inseridos (Inmetro 2018), podendo essa síndrome colaborar com o aparecimento de sintomas como a irritação de olhos, nariz e garganta, fadiga mental, dor de cabeça, tosse, respiração ruidosa, dificuldade em respirar, coceira e hipersensibilidade não específica e dores articulares (Degobbi \& Gambale 2008; Inmetro 2018).

Estudos mostram que a exposição a fungos do ar parece estar associada ao desenvolvimento de várias doenças respiratórias, em especial às asmas ditas "de clima", as quais estão na dependência ou em relação íntima com a flora micótica do ar. Outras patologias tais como aspergilose, rinite alérgica, sinusite fúngica alérgica, asma e alveolite alérgica extrínseca e algumas reações tóxicas também podem ser associadas (Costa \& Machado 2015; Van Leuken et al. 2016).

No tocante ao conhecimento e o monitoramento da qualidade do ar de centros comerciais ainda temos lacunas na literatura, mesmo com o pleno conhecimento que os microorganismos presentes podem atuar como agentes desencadeadores de quadros de hipersensibilidades imediatas, mediante sua ação como aeroalérgenos (Alves et al. 2009).

Dentro desse contexto, o presente trabalho objetivou analisar a diversidade fúngica no ar de dois centros comerciais com climatização natural de referência no município de Fortaleza-CE, 
com intuito de avaliar o ambiente construído, propondo manter o equilíbrio com o bem-estar das pessoas que usam/transitam nesses espaços.

\section{Metodologia}

A pesquisa foi realizada no município de Fortaleza-CE, com clima tropical, quente, com temperatura anual média de $26.5^{\circ} \mathrm{C}$, apresentando historicamente duas quadras climáticas bem definidas, a quadra seca (junho a novembro) e quadra chuvosa (dezembro a maio). Dentro desse município, foram selecionados dois centros comerciais: um Centro Comercial Particular - CCPa e um Centro Comercial Público - CCPu, ambos de climatização natural e de referência ao atendimento municipal, sendo esses os critérios de inclusão dos mesmos na pesquisa.

O CCPa é instituído sob a forma de condomínio edilício, foi inaugurado em 1994, seu espaço físico, constituído de $7.504 .78 \mathrm{~m}^{2}$, dispõe de três pavimentos de shopping, abrigando 206 lojas, com dez andares. Enquanto do CCPu teve sua construção iniciada em 1809, apresenta uma área de $9.690 .75 \mathrm{~m}^{2}$, com 559 boxes, distribuídos em cinco pavimentos.

A participação desses centros comerciais foi oficializada mediante a assinatura de um termo de autorização solicitado através de um consentimento da Instituição para a realização da pesquisa, assegurando-lhes sigilo quanto aos aspectos éticos e razão social.

Para ambos os centros comerciais, os setores analisados foram térreo, segundo andar, terceiro andar e estacionamento, buscou-se analisar os mesmos setores visando fazer comparativos entre os centros comerciais participantes da pesquisa.

Durante o ano 2017, mensalmente, para a coleta de amostras biológicas provenientes do ar foi utilizado o método da sedimentação passiva em placas de Petri de $150 \mathrm{~mm}$ de diâmetro, contendo o meio de cultura Agar Batata Dextrose (Himedia ${ }^{\circledR}$ ). Cada placa foi exposta por 8 horas, das 8:00 às 16:00, sendo colocada a uma altura de $2 \mathrm{~m}$ acima do solo - próximo da área de respiração humana (Pei-Chin et al. 2000).

Durante todo o período de exposição das placas, os setores com climatização natural foram monitorados através de dados fornecidos pela Fundação Cearense de Meteorologia e Recursos Hídricos - FUNCEME.

Findo o período de coleta, as placas de Petri contendo as amostras biológicas foram vedadas e encaminhadas ao Laboratório de Microbiologia da Universidade Estadual do Ceará LAMIC/UECE. As placas de Petri com as amostras de ar permaneceram incubadas a temperatura de $25-27{ }^{\circ} \mathrm{C}$ por até sete dias, realizando-se observações diárias. Todas as colônias foram analisadas macro e micromorfologicamente (Hoog et al. 2000; Sidrim \& Rocha 2004).

A média de Unidades Formadores de Colônias (UFC) fúngicas por metro cúbico (UFC.m ${ }^{-3}$ ) foram calculadas de acordo com as seguintes definições e fórmula (Equação 1) (Bogomolova \& Kirtsideli 2009):

$$
\mathrm{N}=5 \mathrm{a} \times 10^{4}(\mathrm{bt})^{-1}
$$

Onde: $\mathrm{N}=\mathrm{UFC} / \mathrm{m}^{3}$ de ar por ambiente; $\mathrm{a}=$ número de colônias por placa de Petri; $\mathrm{b}=$ superfície da placa de Petri $\left(\right.$ em $\left.\mathrm{cm}^{2}\right) ; \mathrm{t}=$ tempo de exposição (em minutos).

Os dados foram analisados por meio de estatísticas descritivas e percentagem simples. Posteriormente, as médias foram aplicadas ao programa Assist 5.3, no qual relacionou as médias dos centros comerciais.

\section{Resultados e Discussão}

A contagem global por centro comercial em um ano de monitoramento foi de 13.020 UFC.m ${ }^{-3}$ no CGPa e 11.995 UFC.m ${ }^{-3}$ no CCPu. Os locais com maior número de UFC.m ${ }^{-3}$ dos dois centros foram os estacionamentos (3.867 UFC.m ${ }^{-3}$ no CCPU e 3.413 UFC.m $^{-3}$ no CCPa). Já o ambiente com menor contagem de colônias fúngicas foi o terceiro andar do $\mathrm{CCPu}$ com 2.274 UFC.m ${ }^{-3}$. 
No tocante ao menor quantitativo ser proveniente do CCPu (prédio antigo) e maior quantitativo do CCPa (prédio novo) não foi comprovado diferença estatística entre os dados ( $\mathrm{p} \geq$ 0.05), isso se deve possivelmente à qualidade do ar interno estar relacionado a uma combinação de fatores, como as condições ambientais, os materiais e a estrutura do edifício, as atividades desempenhadas dentro de cada setor, o número dos ocupantes e a taxa de renovação de ar (Bluyssen 2009).

Na Tabela 1 está descrita a soma do número de colônias de cada andar nos dois centros, frente a quantidade de transeuntes em cada setor, também não foi constatada nenhuma diferença estatística ( $\mathrm{p} \geq 0.05)$.

Mesmo assim, destaca-se o térreo de ambos os centros, locais onde há maior fluxo de pessoas, sendo os mesmos, ambientes que despontaram como o segundo mais contaminado. Na mesma Tabela observa-se outras características dos ambientes tais como a presença de animais e de unidade alimentar em determinados setores estudados (Tabela 1).

Tabela 1. Caracterização dos setores analisados por UFC.m ${ }^{-3}$, média de transeuntes, detecção de animais e de unidade alimentar em cada centro comercial analisado, município de Fortaleza-CE.

\begin{tabular}{lcccccccc}
\hline \multicolumn{1}{c}{ Andares } & \multicolumn{2}{c}{ UFC.m $^{-3}$} & \multicolumn{2}{c}{$\begin{array}{c}\text { Média diária de } \\
\text { transeuntes }\end{array}$} & \multicolumn{2}{c}{ Presença de animais } & Unidade alimentar \\
& CCPu & CCPa & CCPu & CCPa & CCPu & CCPa & CCPu & CCPa \\
Térreo & 2.888 & 2.932 & 2.000 & 1.000 & Não & Não & Sim & Sim \\
Segundo & 2.581 & 2.881 & 1.500 & 700 & Não & Não & Não & Não \\
Terceiro & 2.275 & 2.771 & 1.300 & 600 & Não & Sim & Não & Sim \\
Estacionamento & 3.868 & 3.414 & 1.600 & 800 & Sim & Não & Não & Não \\
\hline
\end{tabular}

Ainda sobre a relação de transeuntes que passam diariamente pelo CCPu nos quatro setores do estabelecimento (Figura 1), não se constatou relação estatística entre número de transeuntes e quantitativo fúngico ( $p \geq 0.05$ ), mas observou-se que o quantitativo fúngico do segundo e terceiro andares apresentam uma diversidade semelhante com o seu número de transeuntes conforme demonstra a Figura 1.

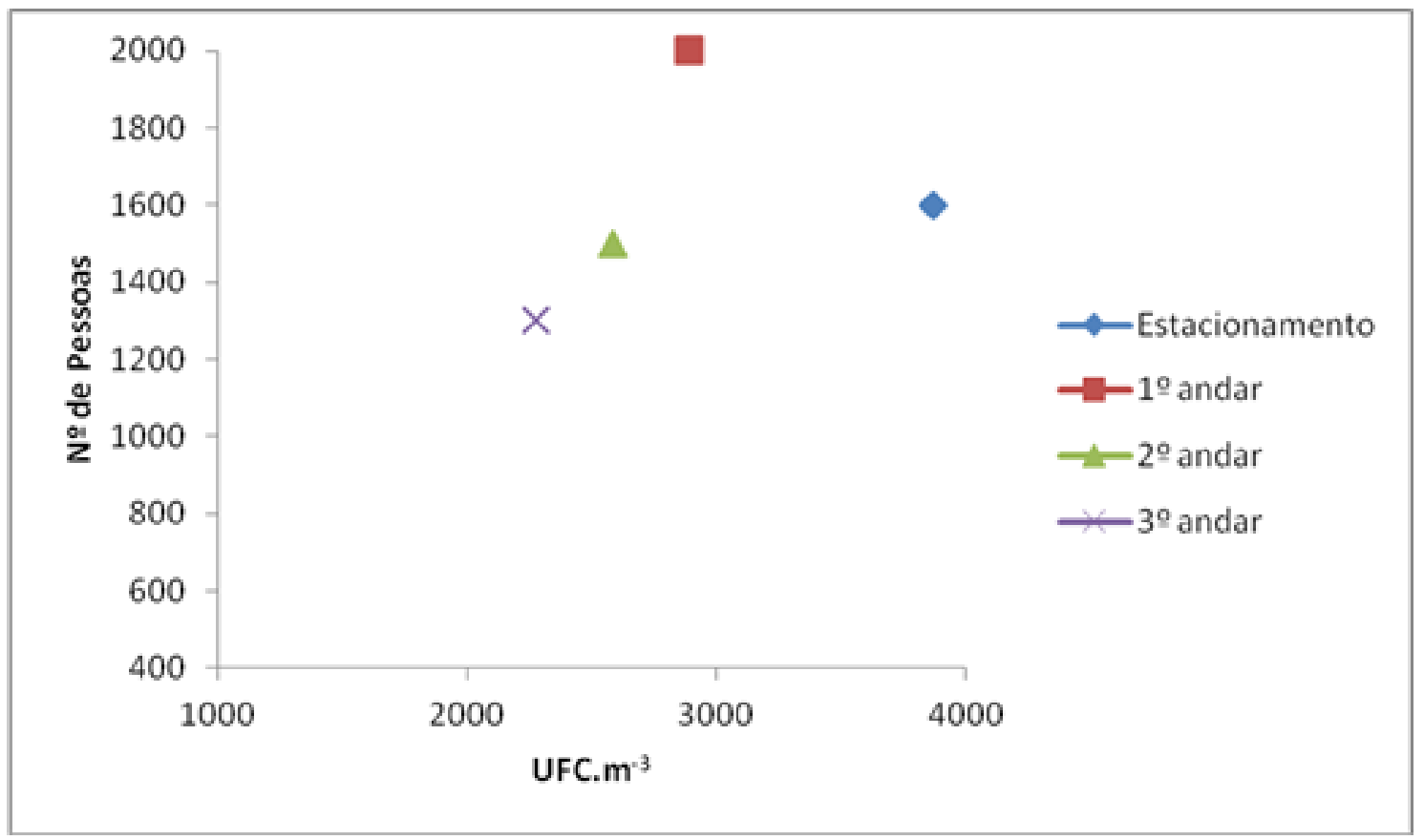

Figura 1. Dispersão da relação do quantitativo fúngico e quantidade de transeuntes em cada setor do Centro Comercial Público $(\mathrm{CCPu})$ monitorado no município de Fortaleza-CE. 
No tocante aos achados do CCPa, a diferença encontra-se apenas no quantitativo de transeuntes, o mesmo caracteriza-se como um estabelecimento de menor número de passantes, entretanto, a diversidade dos dados é semelhante ao CCPu (Figura 2).

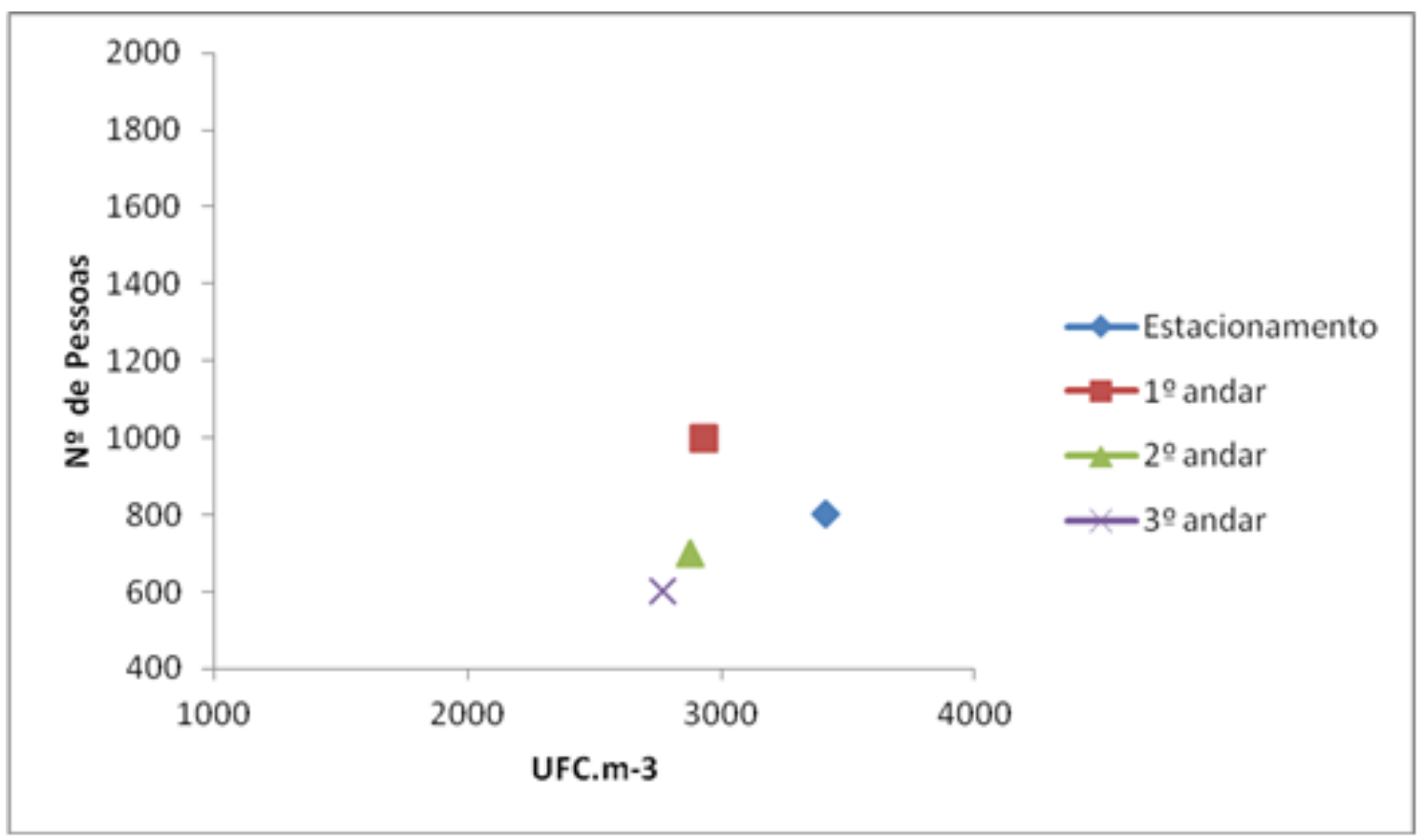

Figura 2. Dispersão da relação do quantitativo fúngico e quantidade de transeuntes em cada setor do Centro Comercial Particular (CCPa) monitorado no município de Fortaleza-CE.

Em ambos os centros comercias os estacionamentos despontaram com os maiores quantitativos fúngicos. Tais achados podem levar há algumas ponderações, uma delas é a pouca renovação de ar, visto que, o estacionamento do CCPa trata-se de um ambiente fechado situado no subsolo, enquanto o estacionamento do CCPu é um espaço coberto, mas revestido de vegetação arbustiva fechada, o que acarreta pouca ventilação ao ambiente, dados que corroboram a pesquisa feita por Henriques (2009), o mesmo afirma que devido ao confinamento do espaço observado em estacionamentos, é necessário, na maioria dos casos, instalar um sistema de ventilação forçada.

Outra possível justificativa é a relação com o número de transeuntes (em ambos os centros, os estacionamentos apresentaram o segundo maior número de passantes). Logo, os locais de elevado fluxo de pessoas em ambos os centros comerciais, como os estacionamentos citados e as praças de alimentação que ficam, em sua maioria, localizadas no térreo, foi averiguado um elevado quantitativo fúngico. Diante disso, os dados apontam que quanto maior a movimentação de pessoas e disponibilidade de substratos orgânicos, pode ter-se um aumento da colonização por fungos.

Esses achados coadunam aos de Teresa et al. (2001), que realizaram pesquisa sobre a concentração microbiana e a influência de alguns fatores ambientais na diversidade de bioaerossóis em cômodos da Faculdade de Ciências Farmacêuticas - UNESP, demonstrando que a concentração desses bioaerossóis em ambientes internos populosos é superior a observada em ambientes onde existe menor circulação de pessoas.

No tocante a diversidade fúngica, foram isolados 28 gêneros fúngicos no CCPa e 22 no $\mathrm{CCPu}$, mesma situação observada frente o quantitativo. Pode-se observar as médias anuais da quantidade de gêneros fúngicos identificados por andar de cada centro comercial, onde é analisado que o térreo do CCPu com o valor de 6.16 gêneros foi maior entre os demais andares dos dois centros, já o terceiro andar do CCPa obteve menor média anual de identificações fúngicas, demonstrando menor diversidade nos achados (Tabela 2). 
Tabela 2. Média anual dos números de gêneros fúngicos encontrados durante as coletas em cada centro comercial monitorado no município de Fortaleza-CE.

\begin{tabular}{lcc}
\hline \multicolumn{1}{c}{ Andar } & Centro comercial público & Centro comercial particular \\
\hline Térreo & 6.16 & 5.09 \\
Segundo andar & 4.58 & 4.66 \\
Terceiro andar & 5.58 & 4.36 \\
Estacionamento & 5.66 & 5.81 \\
\hline
\end{tabular}

No CCPa o gênero Aspergillus foi encontrado em $100 \%$ das amostras, seguido de Penicillium sp., Trichoderma sp., Cladosporium sp., os demais estão citados na Tabela 3. Enquanto, os gêneros isolados no CCPu foram Aspergillus sp., também encontrado em 100\% das amostras, em seguida: Penicillium sp., Fusarium sp., Mucor sp., entre outros (Tabela 3).

Tabela 3. Frequência absoluta da quantidade de vezes que os gêneros fúngicos foram encontrados nos dois centros comerciais monitorados durante o ano de 2017, no município de Fortaleza-CE. Legenda: EST Estacionamento; $1^{\circ}$ AND - $1^{\circ}$ Andar; $2^{\circ}$ AND - $2^{\circ}$ Andar; $3^{\circ}$ AND - $3^{\circ}$ Andar.

\begin{tabular}{|c|c|c|c|c|c|c|c|c|}
\hline \multirow{2}{*}{ Gênero fúngico } & \multicolumn{4}{|c|}{ CG Particular } & \multicolumn{4}{|c|}{ CC Público } \\
\hline & EST & $1^{\circ}$ AND & $2^{\circ}$ AND & $3^{\circ}$ AND & EST & $1^{\circ}$ AND & $2^{\circ}$ AND & $3^{\circ} \mathrm{AND}$ \\
\hline Acremonium & 3 & 2 & 2 & 3 & 3 & 4 & 2 & 3 \\
\hline Alternaria & - & - & - & 3 & 1 & 1 & 1 & - \\
\hline Aspergillus & 12 & 12 & 12 & 12 & 12 & 12 & 12 & 12 \\
\hline Bipolaris & 1 & 2 & - & - & - & 2 & 3 & 1 \\
\hline Cladosporium & 2 & 4 & 4 & 2 & 2 & 2 & 2 & 3 \\
\hline Chrysonilia & 3 & 5 & 2 & 1 & 2 & 1 & 3 & 3 \\
\hline Chrysosporium & 1 & 1 & - & - & 1 & 1 & - & 2 \\
\hline Cladophialophora & 1 & - & - & 1 & 1 & 2 & 2 & 1 \\
\hline Cokeromyces & - & 1 & - & - & - & - & - & - \\
\hline Cunninghamella & 1 & - & - & - & - & - & - & - \\
\hline Curvularia & 1 & - & 2 & 2 & 2 & 1 & 2 & 2 \\
\hline Exophiala & 1 & 1 & - & 1 & - & - & 1 & - \\
\hline Exserohilum & - & - & 1 & - & - & - & - & 1 \\
\hline Fusarium & 2 & 1 & 2 & 1 & 5 & 5 & 3 & 5 \\
\hline Mortierella & 1 & - & - & - & - & - & - & - \\
\hline Mucor & 2 & 4 & 3 & 1 & 4 & 5 & 2 & 4 \\
\hline Nigrospora & 1 & - & - & - & 3 & 1 & - & - \\
\hline Ochoroconis & - & - & 1 & - & - & - & - & - \\
\hline Paecilomyces & 1 & 1 & - & - & - & - & - & - \\
\hline Penicillium & 8 & 6 & 7 & 8 & 8 & 9 & 8 & 9 \\
\hline Phialophora & - & - & - & 1 & - & - & - & - \\
\hline Phoma & - & - & 1 & - & 1 & - & - & - \\
\hline Rhinocladiella & - & - & 1 & - & - & - & - & - \\
\hline Rhizopus & 2 & 3 & 2 & 2 & 3 & 1 & 1 & 1 \\
\hline Rhodotorula & - & - & 1 & - & - & - & - & - \\
\hline Scopulariopsis & - & - & - & 1 & - & 1 & 2 & 1 \\
\hline Scytalidium & 2 & 2 & 2 & - & - & - & 1 & - \\
\hline Trichoderma & 5 & 2 & 4 & 1 & 3 & 3 & - & 5 \\
\hline Trichosporon & 1 & - & 1 & 1 & 1 & 2 & 1 & 1 \\
\hline
\end{tabular}

Dados que não corroboram com estudo realizado na região Nordeste, cuja prevalência foi Cladosporium (42.36\%), seguido do Penicillium (32.73\%), Aspergillus (7.92\%) e Curvularia (7.39\%) como os mais frequentes (Rego \& Santos 2015).

Todos os achados se tratam de fungos com potencial patogênico ao homem, dependendo de condições propícias, como uma baixa imunidade desse hospedeiro. Os achados na presente pesquisa podem estar ligados à gênese de patologias como aspergilose, rinite 
alérgica, sinusite fúngica alérgica, asma e alveolite alérgica extrínseca e algumas reações tóxicas (Costa \& Machado 2015; Van Leuken et al. 2016).

Fungos anemófilos são frequentemente associados a problemas respiratórios, logo, o monitoramento e acompanhamento periódico da qualidade do ar de ambientes internos municia seus gestores de elementos técnicos para aprofundar seus conhecimentos na qualidade do ar, podendo fornecer medidas remediadoras, propondo manter o equilíbrio com o bem-estar das pessoas que usam/transitam no ambiente.

Os valores citados na Tabela 3 representam o número de meses em que ocorreram identificações do gênero fúngico. Com esses achados, os fungos filamentosos hialinos, representados pelos gêneros Acremonium, Aspergillus, Chrysonilia, Fusarium e Penicillium, os zigomicetos Mucor e Rhizopus e o filamentoso demácio Cladosporium estiveram presentes em todos os ambientes, possivelmente motivados por sua ampla distribuição ubíqua.

Estudo realizado em biblioteca pública apresentou também o predomínio de gênero Aspergillus (Pantoja et al. 2012), pesquisa essa realizada utilizando a mesma técnica de sedimentação passiva em placas de Petri com período de exposição de 8 horas.

Outra pesquisa aponta que os gêneros fúngicos mais comuns encontrados no ar são Aspergillus sp., Penicillium sp., Fusarium sp., Cladosporium sp., Curvularia sp., entre outros (Cabral 2010), sendo Aspergillus e Penicillium os primeiros colonizadores de superfícies e interiores (Agarwal \& Chakrabarti 2010), dados que se assemelham a presente pesquisa.

Com base na literatura, a micobiota anemófila pode apresentar variações na dependência da região, devido a fatores como: localidade, estação do ano, entre outros (Flores \& Onofre 2010).

Buscou-se também analisar o quantitativo em UFC.m ${ }^{-3}$ e sua distribuição por estações climáticas (Tabela 4). Utilizou-se o teste T Student para amostras pareadas, não sendo observado diferença significativa $(p \geq 0.05)$ entre a distribuição e quantificação fúngica em relação à sazonalidade.

Tabela 4. Distribuição do quantitativo fúngico $\left(U F C \cdot \mathrm{m}^{-3}\right.$ ) por quadra climática (quadra seca - junho a novembro; e quadra chuvosa - dezembro a maio), com destaque as médias de temperatura, umidade e precipitação atmosférica observadas nos dois centros comerciais monitorados no município de FortalezaCE.

\begin{tabular}{lccccc}
\hline \multirow{2}{*}{ Estações } & \multicolumn{2}{c}{ UFC.m $^{-3}$} & $\begin{array}{c}\text { Temperatura } \\
\left({ }^{\circ} \mathrm{C}\right)\end{array}$ & $\begin{array}{c}\text { Umidade } \\
(\%)\end{array}$ & $\begin{array}{c}\text { Precipitação } \\
(\mathrm{mm})\end{array}$ \\
\hline Seca & CCPu & CCPa & 30.5 & 60.6 & 42.4 \\
Chuvosa & 226.81 & 226.81 & 30.9 & 68.4 & 200.3 \\
\hline
\end{tabular}

Possivelmente isso se deve ao fato de no município de Fortaleza-CE existir ausência de estações climáticas bem definidas, existindo apenas a quadra seca (na qual chove muito pouco) e a quadra chuvosa (com precipitações irregulares que vão de um mínimo de $300 \mathrm{~mm}$ a um máximo de $800 \mathrm{~mm}$ ). Os dados catalogados apontam que a prevalência fúngica independe do período seco e chuvoso, possivelmente devido à pouca variação entre as médias de temperatura $\mathrm{e}$ umidade observada em ambos as quadras (Suliano et al. 2009).

Os resultados registrados nesse trabalho não se assemelham a outros estudos que apontaram existir relação direta entre a contagem das colônias fúngicas com a sazonalidade da região (Teresa et al. 2001; Solomon et al. 2006). Entretanto, corroboram com o trabalho de Pantoja et al. (2012), que não observaram interferência do quantitativo fúngico e as condições climáticas da região cearense. Logo, o monitoramento da qualidade do ar deve ser feito periodicamente para que seja observada alguma variação sazonal com o passar dos anos.

\section{Considerações finais}


$\mathrm{O}$ presente trabalho ao analisar a diversidade fúngica no ar de dois centros comerciais com climatização natural constatou maior quantidade e diversidade fúngica no ambiente particular do que no público, com destaque para os estacionamentos e os andares térreos.

Os fungos Aspergillus e Penicillium foram os mais frequentes, podendo atuar como patógenos primários, mas principalmente como oportunistas, sinalizando a necessidade do uso dos fungos viáveis como marcador epidemiológico da contaminação microbiana, o que poderá contribuir na diminuição de impactos ambientais na vida das pessoas que frequentam e trabalham nesses locais.

Ademais, com o monitoramento e acompanhamento periódico da qualidade do ar de centros comerciais, além de fornecer elementos para aprofundar os saberes sobre esse tipo de espaço, contribui para gerar informações que visam estratégias de prevenção aos possíveis danos causados pelos fungos anemófilos a saúde de seus laborais e traseuntes, assim como, auxiliar na epidemiologia de doenças respiratórias de origem fúngica em centros comerciais.

\section{Referências}

Agarwal R. \& Chakrabarti A. (2010) Epidemiology of allergic bronchopulmonary Aspergillosis (p. 671-688). In: Pasqualotto A.C. (Ed.). Aspergillosis: from diagnosis to prevention. New York: Springer Science. Biomedical and Life Sciences. $1027 \mathrm{p}$.

Alves K.M. da S., Alves A.E.L. \& Silva F.M. (2009) Poluição do ar e saúde nos principais centros comerciais da cidade de Natal/RN. Holos, 4(25): 81-95. DOI: 10.15628/holos.2009.349

Basto J.E. (2007) Qualidade do ar interno. Itajaí, SC, Brasil. [citado 2017/out/14]. Disponível em: http://www.anest.org.br/ (Acessado em 10/09/2019).

Bluyssen P.M. (2009) Towards an integrative approach of improving indoor air quality. Building and Environment, 44: 1980-1989. DOI: 10.1016/j.buildenv.2009.01.012

Bogomolova E. \& Kirtsideli I. (2009) Airborne fungi in four stations of the St. Petersburg Underground railway system. International Biodeterioration and Biodegradation, 63: 156160. DOI: $10.1016 /$ j.ibiod.2008.05.008

Cabral J.P.S. (2010) Can we use indoor fungi as bioindicators of indoor air quality? Historical perspectives and open questions. Science Total Environmental, 408(20): 4285-4295. DOI: 10.1016/j.scitotenv.2010.07.005

Costa G.M.S. \& Machado A.M.B. (2015) Qualidade microbiológica do ar interno de uma creche pública no município de Santa Rita do Sapucaí - MG. Revista Científica da FEPI, 8(especial): $1-10$.

Degobbi C.M. \& Gambale W. (2008) Síndrome dos Edifícios Doentes. Microbiologia in Foco, 4(2): 19-32.

Flores L.H. \& Onofre S.B. (2010) Determinação da presença de fungos anemófios e leveduras em Unidade de Saúde da cidade de Francisco Beltrão-PR. Revista Saúde e Biologia, 5(2): 22-26.

Henriques B.M.S. (2009) Insuflação e extraç̧ão de ar em parques de estacionamento subterrâneo. Dissertação de Mestrado, Programa de Pós-Graduação em Engenharia Mecânica. Faculdade de Ciências e Tecnologia da Universidade Nova de Lisboa. Lisboa, Portugal.

Hoog G.S., Guarro J., Gené J. \& Figueiras M.J. (2000) Atlas of Clinical Fungi. $2^{\circ}$ edition. Baarn/Delft: Centraalbureau voor Schinmelculture/Universitat Rovira i Virgilli. 1126 p.

Inmetro. Instituto Nacional de Metrologia, Qualidade e Tecnologia. (2018) Qualidade do Ar em Estabelecimentos de Uso Público e Coletivo. [citado 2018 mar 18]. Disponível em: http://www.inmetro.gov.br/consumidor/produtos/qualidadedoAr.asp\#normas (Acessado em 10/09/2019).

Pantoja L.D.M., Rizzo R.S., Carvalho B.S., Ferreira V.C., Galas K.S., Fonseca F.R.M. \& Paixão G.C. (2012) Constituição da micobiota aérea de bibliotecas públicas no município de Fortaleza, estado do Ceará, Brasil. Encontros Bibli: revista eletrônica de biblioteconomia e ciência da informação, 17(34): 31-41. DOI: 10.5007/1518-2924.2012v17n34p31 
Pei-Chin W., Huey-Jen S. \& Chia-Yin L. (2000) Characteristics of indoor and outdoor airbone fungi at suburban and urban homes in two seasons. The Science of the Total Environment, 253: 111-118. DOI: 10.1016/S0048-9697(00)00423-X

Pimentel K.S., Souza J.C., Araujo L.M.M., Pantoja L.D.M. \& Paixão G.C. (2016) Influência das atividades humanas na dispersão aérea de fungos demáceos em zona urbana. Ciências \& Tecnologia: FATEC-JB, 8(especial): 1-11.

Quadros M.E. (2008) Qualidade do ar em ambientes internos hospitalares: parãmetros físicoquímicos e microbiológicos. Dissertação de Mestrado, Programa de Pós-Graduação em Engenharia Ambiental. Universidade Federal de Santa Catarina, Florianópolis, Santa Catarina.

Rego C.M. \& Santos F.S. (2015) Ocorrência de fungos anemófilos e sua relação com fatores abióticos em Barreiras, Bahia. Revista Brasileira de Biociências, 13(4): 265-271.

Schossler I., Santana E.R.R. \& Spinelli R. (2015) Avaliação de qualidade do ar interior em relação às características de ventilação das edificações. Revista Destaques Acadêmicos, 7(4): 230-243.

Sidrim J.J.C. \& Rocha M.F.G. (2004) Micologia Médica à Luz de Autores Contemporâneos. Rio de Janeiro: Guanabara Koogan. 476 p.

Solomon G.M., Koski M.H., Ellman M.R. \& Hammond S.K. (2006) Airborne mold and endotoxin concentrations in New Orleans, Louisiana, after flooding, October through November 2005. Environ Health Perspect, 114(9): 1381-1386. DOI: 10.1289/ehp.9198

Statholoupou I., Assimakopoulos V.D., Flocas V.A. \& Helmis C.G. (2008) An experimental study of air quality inside large atheletichalls. Building an Enviromment, 43(5): 793-803. DOI: 10.1016/j.buildenv.2007.01.026

Suliano D.C., Magalhães K.A. \& Soares R.B. (2009) A influência do clima no desempenho da economia cearense. Textos para Discussão do Instituto de Pesquisa e Estratégia Econômica do Ceará (IPECE). Disponível em: https://www.ipece.ce.gov.br/wp-content/uploads/sites/45/2014/0 2/TD_56.pdf (Acesso em 02/08/2019).

Sunyer J., Suades-González E., García-Esteban R., Rivas I., Pujol J., Alvarez-Pedrerol M., Forns J., Querol X. \& Basagañaa X. (2017) Traffic-related Air Pollution and Attention in Primary School Children. Epidemiology, 28(2): 181-189. DOI: 10.1097/EDE.0000000000000603

Teresa D.B., Ponsoni K. \& Raddi M.S.G. (2001) Bioaerossóis em Ambientes do Prédio Tradicional da Faculdade de Ciências Farmacêuticas - UNESP. Revista de Ciências Farmacêuticas, 22(1): 31-39.

Van Leuken J.P.G., Swart A.N., Droogers P., Pul A., Heederik D. \& Havelaar A.H. (2016) Climate change effects on airborne pathogenic bioaerosol concentrations: a scenario analysis. Aerobiologia, 32: 607-617. DOI: 10.1007/s10453-016-9435-5 